\title{
Gravitational light deflection, time delay and frequency shift in Einstein-Aether theory
}

\author{
Kai Tang ${ }^{1,2}$, Tian-Yi Huang ${ }^{3}$, Zheng-Hong Tang ${ }^{1}$ \\ ${ }^{1}$ Shanghai Astronomical Observatory, Chinese Academy of \\ Sciences;tangkai@shao.ac.cn;zhtang@shao.ac.cn \\ ${ }^{2}$ Graduate School of Chinese Academy of Sciences \\ ${ }^{3}$ Department of Astronomy, Nanjing University;tyhuang@nju.edu.cn
}

\begin{abstract}
Einstein-Aether gravity theory has been proven successful in passing experiments of different scales. Especially its Eddington parameters $\beta$ and $\gamma$ have the same numerical values as those in general relativity. Recently Xie and Huang (2008) have advanced this theory to a second post-Newtonian approximation for an N-body model and obtained an explicit metric when the bodies are point-like masses. This research considers light propagation in the above gravitational field, and explores the light deflection, time delay, frequency shift etc. The results will provide for future experiments in testing gravity theories.
\end{abstract}

Keywords. Einstein-Aether, light deflection, time delay, frequency shift

\section{Introduction}

Einstein-Aether theory is a vector-tensor theory. It postulates a dynamical unit timelike vector field besides the metric [1]. This vector can be viewed as the minima structure required to determine a local preferred rest frame. We call it the "aether" as it is ubiquitous and determines a locally preferred frame at every point of space-time [2].

Recently, Xie and Huang (2008) have advanced this theory to a second post-newtonian approximation for a N-body model and obtained an explicit metric when the bodies are point-like masses [3]. In this research, we only consider the case that light closely passes the limb of the sun and neglect the contributions from planets. And the metric in isotropic gauge for such a practical $2 \mathrm{PN}$ light deflection experiments can be simplified as

$$
\begin{aligned}
\mathrm{d} s^{2} & =\left(-1+\frac{2 G M}{c^{2} r}-\frac{2 G^{2} M^{2}}{c^{4} r^{2}}\right) c^{2} \mathrm{~d} t^{2} \\
& +\left(1+\frac{2 G M}{c^{2} r}+\frac{3}{2} \frac{G^{2} M^{2}}{c^{4} r^{2}}+\frac{1}{4} c_{14} \frac{G^{2} M^{2}}{c^{4} r^{2}}\right)\left(\mathrm{d} r^{2}+r^{2} \mathrm{~d} \theta^{2}+r^{2} \sin ^{2} \theta \mathrm{d} \phi^{2}\right),
\end{aligned}
$$

which is quite close to that adopted by Brumberg (1991) [4], Teyssandier and Le PoncinLafitte (2008) [5] and Klioner and Zschocke (2009) [6].

There is a $2 \mathrm{PN}$ parameter $c_{14}$ which is the only non-GR parameter remaining as the deviation from general relativity. It shows the difference between these two gravity theories. The measurement of $c_{14}$ can be achieved in future experiment with light propagating in the field of the Sun.

Since the field is isotropic, we may consider the space track of the light to be confined to the equatorial plane, that $\theta=\frac{\pi}{2}$. And we define the coordinate system as shown in Figure 1. The origin $O$ is the mass center of the Sun. $D$ is the nearest point to the Sun in the light propagation, $\mathbf{O D}$ is the pole axis. And the light ray propagates between two 


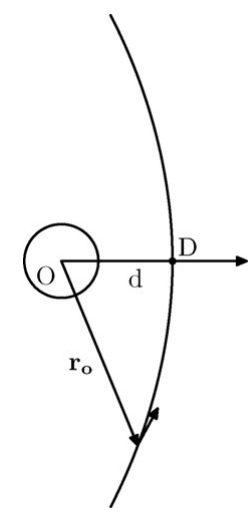

Figure 1. Light propagation in the gravitational field of the Sun

points, being emitted at a position $\mathbf{r}_{\mathbf{s}}\left(r_{s}, \varphi_{s}\right)$ at the time moment $t_{s}$ and received at a position $\mathbf{r}_{\mathbf{o}}\left(r_{o}, \varphi_{o}\right)$ at the time moment $t_{o}$.

\section{Light propagation}

Metric(1.1) does not depend explicitly on $t$ and $\phi$, so we can make use of two invariants to simplify our derivation. And the space track of light in our isotropic spherical coordinates is as follows,

$$
\begin{aligned}
\varphi & =\arccos \frac{d}{r}+\frac{2 G M}{c^{2} d} \sqrt{\frac{r-d}{r+d}} \\
& +\frac{G^{2} M^{2}}{c^{4}}\left[-\frac{2(3 d+2 r) \sqrt{r^{2}-d^{2}}}{(d+r)^{2} d^{2}}+\frac{15}{4 d^{2}} \arccos \frac{d}{r}+\frac{c_{14}}{8 d^{2}} \arccos \frac{d}{r}\right],
\end{aligned}
$$

in which $d$ is the closest distance of the light path from the Sun.

For testing these two gravity theories, we obtain the post-post-Newtonian equation of light deflection, time delay and frequency shift in a static gravitational field from the $\operatorname{metric}(1.1)$.

\section{Light Deflection}

When the source is at infinity, we found the angle between the observed and the coordinate direction of the source, which is the light deflection, as the following,

$$
\begin{aligned}
& \frac{2 G M}{c^{2} d}\left(1+\frac{\sqrt{r_{o}^{2}-d^{2}}}{r_{o}}\right)+\frac{G^{2} M^{2}}{c^{4}}\left[\frac{15 \pi}{8 d^{2}}-\frac{4}{d^{2}}-\frac{\sqrt{r_{o}^{2}-d^{2}}\left(d^{2}+d r_{o}+16 r_{o}^{2}\right)}{4 r_{o}^{2}\left(d+r_{o}\right) d^{2}}+\frac{15}{4 d^{2}} \arccos \frac{d}{r_{o}}\right] \\
& +\frac{G^{2} M^{2}}{c^{4}}\left[\frac{c_{14}}{8 d^{2}} \arccos \frac{d}{r_{o}}+\frac{c_{14} \pi}{16 d^{2}}+\frac{c_{14} \sqrt{r_{o}^{2}-d^{2}}}{8 d r_{o}^{2}}\right],
\end{aligned}
$$

in which

$$
d=r_{0} \sin \beta-\frac{2 G M}{c^{2}}(1-\sin \beta)
$$

where $\beta$ is the angle between the observed direction of the source and $\mathbf{r}_{\mathbf{o}}$. 
2. Time Delay

We denote $\mathbf{R}=\mathbf{r}_{\mathbf{s}}-\mathbf{r}_{\mathbf{o}}, R=|\mathbf{R}|$. The formula for the time of light propagation is

$$
\begin{aligned}
& R+\frac{2 G M}{c^{2}} \ln \frac{r_{s}+r_{o}+R}{r_{s}+r_{o}-R}+\frac{G^{2} M^{2}}{c^{4}} \frac{2 R}{\left|\mathbf{r}_{o} \times \mathbf{r}_{s}\right|^{2}}\left[\left(r_{o}-r_{s}\right)^{2}-R^{2}\right] \\
& +\frac{G^{2} M^{2}}{c^{4}} \frac{R}{\left|\mathbf{r}_{o} \times \mathbf{r}_{s}\right|}\left(\frac{15}{4} \arccos \frac{r_{o}^{2}+r_{s}^{2}-R^{2}}{2 r_{s} r_{o}}+\frac{c_{14}}{8} \arccos \frac{r_{o}^{2}+r_{s}^{2}-R^{2}}{2 r_{s} r_{o}}\right) .
\end{aligned}
$$

When $c_{14}=0$, it's the same as the result previously derived by different approaches [4] [5].

3. Frequency Shift

The observed gravitational shift of frequency is

$$
1+z=\frac{\mathrm{d} \tau_{s}}{\mathrm{~d} \tau_{o}}=\frac{\mathrm{d} \tau_{s}}{\mathrm{~d} t_{s}} \frac{\mathrm{d} t_{s}}{\mathrm{~d} t_{o}} \frac{\mathrm{d} t_{o}}{\mathrm{~d} \tau_{o}},
$$

where

$$
\begin{gathered}
\frac{\mathrm{d} \tau_{s}}{\mathrm{~d} t_{s}}=1-\frac{G M}{c^{2} r_{s}}-\frac{\dot{\mathbf{r}}_{s}^{2}}{2 c^{2}}+\frac{G^{2} M^{2}}{2 c^{4} r_{s}^{2}}-\frac{3 G M}{2 c^{2} r_{s}} \frac{\dot{\mathbf{r}}_{s}^{2}}{c^{2}}-\frac{1}{8} \frac{\dot{\mathbf{r}}_{s}^{4}}{c^{4}} \\
\frac{\mathrm{d} t_{o}}{\mathrm{~d} \tau_{o}}=1+\frac{G M}{c^{2} r_{o}}+\frac{\dot{\mathbf{r}}_{o}^{2}}{2 c^{2}}+\frac{G^{2} M^{2}}{2 c^{4} r_{o}^{2}}+\frac{5 G M}{2 c^{2} r_{o}} \frac{\dot{\mathbf{r}}_{o}^{2}}{c^{2}}+\frac{3}{8} \frac{\dot{\mathbf{r}}_{o}^{4}}{c^{4}} \\
\frac{\mathrm{d} t_{s}}{\mathrm{~d} t_{o}}=\frac{1-A \frac{\mathbf{R} \cdot \dot{\mathbf{r}}_{o}}{c R}+B \frac{\mathbf{r}_{o} \cdot \dot{\mathbf{r}}_{o}}{c r_{o}}+D \frac{\left(\mathbf{r}_{o} \times \mathbf{r}_{s}\right) \cdot\left(\dot{\mathbf{r}}_{o} \times \mathbf{r}_{s}\right)}{c\left|\mathbf{r}_{o} \times \mathbf{r}_{s}\right|}}{1-A \frac{\mathbf{R} \cdot \dot{\mathbf{r}}_{s}}{c R}-C \frac{\mathbf{r}_{s} \cdot \dot{\mathbf{r}}_{s}}{c r_{s}}-D \frac{\left(\mathbf{r}_{o} \times \mathbf{r}_{s}\right) \cdot\left(\mathbf{r}_{o} \times \dot{\mathbf{r}}_{s}\right)}{c\left|\mathbf{r}_{o} \times \mathbf{r}_{s}\right|}}
\end{gathered}
$$

with $\dot{\mathbf{r}}_{o}=d \mathbf{r}_{o} / d t_{o}$ and $\dot{\mathbf{r}}_{s}=d \mathbf{r}_{s} / d t_{s}$,

$$
\begin{aligned}
A=1 & +\frac{4 G M}{c^{2}} \frac{r_{o}+r_{s}}{\left(r_{o}+r_{s}\right)^{2}-R^{2}}+\frac{2 G^{2} M^{2}}{c^{4}} \frac{\left(r_{o}-r_{s}\right)^{2}-3 R^{2}}{\left|\mathbf{r}_{o} \times \mathbf{r}_{s}\right|^{2}} \\
+ & \frac{\left(\frac{15}{4}+\frac{c_{14}}{8}\right)}{\left|\mathbf{r}_{o} \times \mathbf{r}_{s}\right|} \frac{G^{2} M^{2}}{c^{4}}\left[\arccos \frac{r_{o}^{2}+r_{s}^{2}-R^{2}}{2 r_{s} r_{o}}+\frac{2 R^{2}}{\sqrt{\left(r_{o}+r_{s}\right)^{2}-R^{2}} \sqrt{R^{2}-\left(r_{o}-r_{s}\right)^{2}}}\right] \\
B= & -\frac{4 G M}{c^{2}} \frac{R}{\left(r_{o}+r_{s}\right)^{2}-R^{2}}+\frac{4 G^{2} M^{2}}{c^{4}} \frac{R\left(r_{o}-r_{s}\right)}{\left|\mathbf{r}_{o} \times \mathbf{r}_{s}\right|^{2}} \\
& -\left(\frac{15}{4}+\frac{c_{14}}{8}\right) \frac{G^{2} M^{2}}{c^{4}} \frac{R}{\left|\mathbf{r}_{o} \times \mathbf{r}_{s}\right|} \frac{r_{o}^{2}+R^{2}-r_{s}^{2}}{\left.r_{o}+r_{s}\right)^{2}-R^{2}} \sqrt{R^{2}-\left(r_{o}-r_{s}\right)^{2}} \\
C= & -\frac{4 G M}{c^{2}} \frac{4 G^{2} M^{2}}{\left(r_{o}+r_{s}\right)^{2}-R^{2}}+\frac{R\left(r_{s}-r_{o}\right)}{\left|\mathbf{r}_{o} \times \mathbf{r}_{s}\right|^{2}} \\
& -\left(\frac{15}{4}+\frac{c_{14}}{8}\right) \frac{G^{2} M^{2}}{c^{4}} \frac{R}{\left|\mathbf{r}_{o} \times \mathbf{r}_{s}\right|} \frac{r_{s}^{2}+R^{2}-r_{o}^{2}}{r_{s} \sqrt{\left(r_{o}+r_{s}\right)^{2}-R^{2}} \sqrt{R^{2}-\left(r_{o}-r_{s}\right)^{2}}} \\
D= & -\frac{4 G^{2} M^{2}}{c^{4}} \frac{R\left[\left(r_{o}-r_{s}\right)^{2}-R^{2}\right]}{\left|\mathbf{r}_{o} \times \mathbf{r}_{s}\right|^{3}}-\left(\frac{15}{4}+\frac{c_{14}}{8}\right) \frac{G^{2} M^{2}}{c^{4}} \frac{R \arccos \frac{r_{o}^{2}+r_{s}^{2}-R^{2}}{2 r_{s} r_{o}}}{\left|\mathbf{r}_{o} \times \mathbf{r}_{s}\right|^{2}}
\end{aligned}
$$




\section{Discussion}

The analytical solution for light deflection, time delay and frequency shift is derived in this note. The terms containing $c_{14}$ are the main difference between general relativity and Einstein-Aether theory. This result can be provided for future experiments in testing gravity theories. Klioner and Zschocke have gotten the time delay in harmonic gauge [6], and the result we got is in isotropic condition. The comparison of the two works can be achieved through a coordinate transformation. The transformation is the Formula 129 given in [3]. Detailed procedure of derivation will be given elsewhere.

\section{Acknowledgement}

The authors thank the editor and referee for their kind comments. This work is supported by the Natural Science Foundation of China under the Grant Nos. 10673026 and 10873014.

\section{References}

[1] Jacobson, T. \& Mattingly, D. 2001, Phys.Rev.D, 64, 024028

[2] Jacobson, T. \& Mattingly, D. 2004, Phys.Rev.D, 70, 024003

[3] Xie, Y. \& Huang, T.-Y. 2008, Phys.Rev.D, 77, 124049

[4] Brumberg, V. A. 1991, Bristol, England and New York, Adam Hilger, 1991, 271 p.,

[5] Teyssandier, P. \& Le Poncin-Lafitte, C. 2008, Classical and Quantum Gravity, 25, 145020

[6] Klioner, S. A. \& Zschocke, S. 2009, arXiv:0902.4206 